\title{
Foregoing prehospital care: should ambulance staff always resuscitate?
}

\author{
Kenneth V Iserson The University of Chicago
}

\section{Author's abstract}

Approximately 400,000 people die outside US hospitals or chronic care facilities each year. While there has been some recent movement towards initiating procedures for prehospital Do Not Resuscitate (DNR) orders, the most common situation in the US is that emergency medical systems (EMS) personnel are not authorised to pronounce patients dead, but are required to attempt resuscitation with all of the modalities at their disposal in virtually all patients. It is unfair and probably unrealistic for EMS personnel to have to make a determination of the validity of a non-standard prehospital DNR order (for example, a living will or a durable power of attorney for health care). Existing prehospital DNR protocols range from being very restrictive in the scope of patients allowed to participate and in their implementation, to those that are more liberal. Potential benefits of prehospital DNR orders include freeing up vital personnel and material for use by those who would more fully benefit, and alleviating the enormous emotional strain on patients, families, EMS personnel, and hospital medical staffs involved in unwanted resuscitations that only prolong the dying process. Given this, prehospital DNR orders present several legal and moral problems. These include proper patient identification, the nature of the document itself, precautions incorporated into a DNR system to prevent misuse, potential liability for EMS and hospital personnel, and potential errors in implementation. Functioning prehospital DNR systems need to include: 1) specific legislation detailing the circumstances in which such a document could be used, the wording of such a document, and protection from liability for those implementing the document's directives; 2) having the currently valid document immediately available to the EMS personnel or base station doctors; and 3) acceptable means of identifying the patient. Relatively few US jurisdictions as yet have a prehospital DNR order system, although it is an idea whose time is overdue. Society's imperative to use available technology has pushed us into a situation where a technique to save those with a potential to continue a meaningful and

\section{Key words}

Death; dying; DNR orders. wanted existence is being used indiscriminately to prolong the agony of death.

Approximately 400,000 people die outside hospitals or chronic care facilities each year in the Untied States $(1,2)$. All of them have a cessation of heart activity, ie, cardiac arrest, which is the final common pathway for all deaths. Cardiopulmonary resuscitation (CPR) along with adjunctive procedures are generally considered the treatment for cardiac arrest. But, according to the National Conference on Cardiopulmonary Resuscitation and Emergency Cardiac Care, CPR was initially designed to prevent 'sudden unexpected death ... [and was] not indicated in certain situations, such as in cases of terminal irreversible illness where death [was] not unexpected' (3). However, there is often scant information with which to discriminate amongst the prehospital patients in whom resuscitation should and should not be attempted. This frequently results in a dismal rate of survival (4). Many of the patients for whom resuscitation is performed have a minimal ability to function or ultimately survive, and perhaps should not have had any resuscitative efforts performed at all (5). The American Heart Association (AHA) states that in hospital settings, 'it was not too long [after the introduction of CPR techniques] before CPR was performed almost reflexly, so much so that a mechanism had to be developed to prevent resuscitative efforts in patients who were hopelessly ill. "Do Not Resuscitate" (DNR) or "Care and Comfort Only" orders were invoked' (6). However, currently most US emergency medical systems (EMS) do not easily allow this option $(4,7,8,9)$.

Prehospital care is simplistically defined as that care delivered prior to the patient's arrival at an acute care medical facility. The entire prehospital medical care arena includes everywhere outside of the emergency department: the home, long-term care facility, hospice, and community. If all of these sites are included, the number of deaths occurring outside hospitals and chronic care facilities is even greater than the 400,000 cited above. For acute medical care, especially resuscitations with intravenous drugs, electrical defibrillation and pacing of the heart, and intubation of the airway, the entire prehospital care 
arena is served by EMS workers, ie, paramedics. With the increased sophistication of the US EMS in recent years, ambulance crews dispatched by the elegant 911 telephone network are capable of delivering advanced life support techniques which were not available in many hospitals until recently. These EMS personnel are, however, neither nurses nor doctors, have only a smattering of formal training in ethical issues, and must usually operate under a strict set of protocols set up by the state or local EMS governing body (10). This limits their options and leads to situations where intensive critical care procedures must be used, by protocol, on out-of-hospital patients in extremis or in cardiac arrest any time the ambulance service is activated (9).

A typical case, drawn from the multitude of similar cases that the author has seen over the years, is that of a 45-year-old woman who, the previous week, had been discharged home from the hospital with a diagnosis of terminal metastatic pancreatic cancer. Both she and her family were comfortable with the decision they had made (and discussed with their primary care doctor) to forego further treatment and to allow her to die peacefully at home. However, when she lapsed into coma and had obvious respiratory distress, her husband felt helpless and in need of assistance. Not being able to quickly contact her doctor, he called the 911 emergency telephone number. The paramedic ambulance crew arrived, recognised the severity of the acute condition and begun resuscitative procedures. The woman had an endotracheal tube positioned to breathe for her, an intravenous line to administer medications, and cardiac monitor leads placed to watch for arrhythmias. Although the husband protested that his wife was terminal and did not wish this care, his pleading fell on deaf ears. The woman was transported to the local emergency department where the care was continued. Only then, after her identity could be confirmed, and her records and physician located, could intensive lifesustaining care be halted and the woman allowed to die. This care tied up an advanced life support ambulance team for one hour, and the hospital emergency care personnel for nearly as long. The cost, in a typical mid-western US city, would be about $\$ 1,300$. And, while the woman was never aware of her mode of death, her family, the ambulance crew, the hospital emergency medical team, and her personal doctor were all distressed. If the patient were to be 'successfully' resuscitated to a state of sentience, the moral tragedy would be compounded by her additional pain and suffering accompanying the dying process for the second time (11).

This case illustrates a common scenario in the United States. While there has been some recent movement towards initiating procedures for prehospital DNR orders, at present the most common situation nationwide is that prehospital care providers must provide full resuscitative care for all patients in cardiac or respiratory arrest for whom they are called
(7,8,12-19). A DNR order, in the current medical vernacular, is a request for providers of medical care not to initiate lifesaving measures. At the least, it includes a request not to perform cardiopulmonary resuscitation. The other elements of such orders can vary, but will often include a request not to intubate a patient, not to place the patient on a ventilator, nor to electrically shock the heart.

Occasionally, it will also include a request to avoid using parenteral medications, hydration, or other measures sometimes considered basic medical support. Perhaps, given the extensive nature of some of these DNR orders, they might better be termed 'No Extraordinary Care' orders. This term at least implies that the exact meaning of the order must be discussed with the patient or, if incompetent, with the legal guardian or family, to be certain exactly what is meant, and to ensure that it is fully and accurately described.

Requests for DNR orders have, in the past, often been initiated by clinicians - either with or without the knowledge of patients or the family (9). With more public awareness of the available options, patients and families themselves are now initiating discussions of DNR orders with clinicians. This is often done in the guise of discussing living wills or durable powers of attorney for health care. Yet even when primary care providers have discussed methods of death with their patients, they still often feel impotent due to their inability to stop their patients' resuscitation by the emergency medical care system once the patient is out of the hospital. On the other hand, the experiences of emergency medicine, critical care, and other hospitalbased clinicians is that they are often left with difficult decisions when patients, apparently known to have been terminal and rapidly deteriorating, are hospitalised without any prior discussions with the patient or decisions regarding the aggressiveness of care. This indicates that there are still many primary care clinicians who, for emotional or other reasons, are reluctant to discuss options for the methods of death with their patients. With the wide publicity given to Kubler-Ross's work on death, the President's Commission reports, and the subsequent increased education on death in medical schools, it was widely assumed that clinicians would be more open to discussing the subject of death with their patients (2022). This apparently was incorrect (23). Although not a traditional role, it may fall to social service, religious, or nursing personnel to initiate such discussions with patients.

Who can execute a DNR order? This is an interesting question. Cardiopulmonary resuscitation is the only medical procedure that virtually anyone can perform, yet once started it needs a doctor's order, in the medical institution or EMS, to stop. For nonmedical personnel who begin CPR, the AHA recommends 'that the non-physician continue basic life support as long as it is needed or until the rescuer is exhausted or relieved by a physician, another rescuer, or a rescue team' (6). Similarly, an order not to 
begin resuscitative procedures within a health care facility (a DNR order) must be executed by a licensed doctor. In general, it must be with the consent of the patient, a legal guardian, legally approved family members, or the court; the specific individuals who may give consent to a DNR order for an incompetent patient varies by jurisdiction. Yet, in critical situations of acute onset, except for the parent-minor child relationship, previously established legal dependency is rarely seen. And the relatives who are informed of, and are frequently asked to consent to, procedures, are often not actually legally able to do so. Therefore, they certainly are not able to refuse such potentially lifesaving procedures as resuscitation; such a refusal may be predicated on the emotional or financial burden produced by a prolonged illness $(24,25)$.

Many teaching institutions now require that attending doctors, rather than residents, sign DNR orders. Recently, some institutions have begun to use specific forms for DNR orders in order to identify the elements, such as appropriate discussions with the patient or surrogate, necessary to make them valid. A durable power of attorney, with definite provisions for a specific individual to make medical decisions if the patient is unable to make his or her own decisions, might also allow consent for a DNR order. If the DNR request is contained within a living will, it is executed under state law, by the patient.

How do prehospital DNR orders work? The most elegant place at which effectively to utilise a prehospital DNR order is at the step of activating the 911 or equivalent local ambulance dispatch system. In one California EMS system, individuals who have a prehospital DNR order on record are logged into a computer. If the name matches when the paramedics call in by radio, or if it matches the name the dispatchers receive at the time of the initial call, the paramedics are advised of this and they do not perform or they halt resuscitation efforts, if the patient is either wearing an official 'DNR' name tag or the prehospital DNR document is present (16). Ideally, this could work in one of two ways depending upon where the patient resides. If the patient is in a long-term health care facility, there could be a permanent DNR order written to prevent the staff from either beginning resuscitative procedures or calling for an ambulance. The order, however, would have to be prominently displayed and evident to all, including the commonly used per diem or agency staff nurses and aides. It has been suggested that a nursing home DNR order be valid for two years (8). Due to the chronicity and inevitability of the disease process in long-term nursing home patients, this seems to be a reasonable approach. On the other hand, if the patient resides at home, optimally the family would not call for ambulance assistance when the patient deteriorated. But this can be difficult in a culture where a familiarity with death at home is no longer common. One benefit of executing a prehospital DNR order may be an opportunity to discuss with the clinician the exact procedures to be followed by the family around the time of death.

If the ambulance system is activated, the problems multiply. In most states, EMS personnel are not authorised to pronounce patients dead. They are required to attempt resuscitation with all of the modalities at their disposal except in the most obvious cases of death. At present, most EMS systems recognise 'obvious death' in most patients with evidence of decomposition, dependent lividity, decapitation, incineration, or massive injury to the heart, lungs, or brain that is incompatible with life (10). Of course a patient with appropriate decisionmaking capacity can refuse medical care from either doctors or EMS personnel, as long as the counterbalancing state interests (1. preservation of life; 2. prevention of homicide and suicide; 3. protection of interests of innocent third parties; 4. preservation of the integrity of the medical profession) are not threatened (26). This right has been repeatedly upheld in the courts both on the basis of a guaranteed right to privacy and a liberty interest found in the US Constitution $(27,28)$. However, a patient who does not have this capacity gives implied consent to emergency treatment if no acceptable advance directive is present. 'Acceptable' in this sense means a DNR order for which the EMS personnel have been trained and which is applicable to the pre-hospital setting.

In the often frenetic scene surrounding a prehospital cardiac arrest, it is unfair and probably unrealistic for EMS personnel to have to make a determination of the validity of a non-standard prehospital DNR order. This includes limited DNR orders, such as 'do not intubate' or 'do not shock'. In most situations, even if a DNR order, other than a standard and accepted prehospital DNR order exists, ambulance crews would not be expected to alter their care. The National Association of Emergency Medical Services Physicians (NAEMSP), in a document advocating adoption of prehospital DNR protocols, states that 'the prehospital provider must always presume in favour of performing cardiopulmonary resuscitation in cases where a legallyvalid "Do Not Resuscitate" order is not in place. The chaos of an emergency call is not the place for making "Do Not Resuscitate" decisions or evaluating the validity of a "Do Not Resuscitate" order. Most living wills have some conditions that must be met prior to the will being in effect. It is not always clear to the Emergency Medical Technician (EMT) that such conditions have been met at the time of being presented with such a document. Finally, it is far beygnd the training of an EMT upon being presented with a living will to determine its legal validity' (29). It has been suggested though, that 'confusion is minimised at the scene when there is a clear understanding by the medical community of the proper format for communicating resuscitation status and the treatments that are appropriate and should be provided when indicated' (30).

Existing prehospital DNR protocols range from 
being very restrictive in the scope of patients allowed to participate and in their implementation (13), to those that are more liberal $(16,17)$. Maryland's protocol, for example, requires patients to have a terminal illness, a life expectancy of six months or less, and be under the care of a hospice programme (but not necessarily in the hospice). A 'hospice card' with detailed information must be with the patient when the paramedics arrive, and a separate confirmation of identity is required (13). On the other hand, the Anchorage, Alaska protocol is effective for everyone except those in long-term facilities (for legal reasons) and is very simple to implement. A special phone number is reserved for the family to call at the time of death (17).

If these types of prehospital DNR systems are not in place, the patient will then be transported to an emergency department. Emergency physicians, with their commitment to universal care for life-threatening conditions and the time constraints necessary to perform a successful resuscitation, are ill-equipped initially to assess the validity of a non-prehospital advance directive handed them by ambulance personnel or family members. Only when the resuscitative process has been terminated due to success or death, can the pace be slowed enough to begin to evaluate whether a legal document can or should affect the treatment of a specific patient. This conforms to a universal rule in health law, 'treat first and ask legal questions later' (31). And in fact, it will probably fall to the critical care doctor in the intensive care unit, with the help of a hospital attorney, to evaluate such a document. If it is found to be valid, life support could then be withdrawn - but the added pain, expense, and anguish would already have occurred.

What are the potential benefits of prehospital DNR orders? If utilised appropriately, these orders can limit the utilisation of scarce EMS and hospital resources by individuals who do not want and will not benefit from resuscitation. This will free vital personnel and material for use by those who would more fully benefit. These dedicated professionals, whose job is daily to bring back those on the edge of death, undergo an enormous emotional strain when it is clear that their efforts are not only useless, but distasteful to the patient with whom they are working. This was a specific reason for implementing the Anchorage, Alaska protocol (17). They noted that 'emotional risk is an occupational hazard in EMS, so much so that actual grief reactions on the part of the EMT occur with distressing frequency. Ironically, the sense of personal loss linked to certain calls does not coincide with patient outcome. Successfully reviving a patient who is known to be terminally ill, or survives to endure an empty existence, can cost the responder much more emotionally than losing the patient ... Failure to grasp and fulfill our patients' needs deprives us of one of the sustaining rewards of EMS work' (32). Prehospital DNR orders would lessen the number of patients, families, and clinicians placed in this scenario. It would also have the effect of avoiding the pain and anguish of both the patient's family and the patient who didn't want to be resuscitated. The Alaskans also noted that prehospital DNR protocols had as '... a valuable sideeffect the potential reduction in several intangible costs that accompany these calls: conflicts at the scene, skewed reponse patterns, and self-esteem decay surrounding treatment of hopelessly ill patients' (32).

What are the legal and moral problems that prehospital DNR orders present? The most obvious problem of actually employing DNR orders originating in the prehospital arena, is patient identification. When individuals are hospitalised, they are under a defined identification system, they are known to the staff who are responsible for activating the resuscitation team, and there is a defined method of writing and accepting medical orders. To EMS personnel, the identification of patients in the prehospital care system is often less certain. The more serious the patient's condition or the more confused the setting in which the patient is located, the more difficult it may be positively to identify the patient. This is implicitly recognised by the common use of coded names and numbers in most emergency departments to identify critical patients until a positive identification can be made. The systems that have adopted successful prehospital DNR systems have implicitly or explicitly relied upon the concept of a 'good faith effort' on the part of those in attendance to identify the patient (13-19). In writing recently about prehospital DNR systems, one doctor noted that 'a critical component of a DNR system is ensuring the validity of a DNR order by using the medical record and physician signatures or using standardised forms and a system of validation' (30). While this might not meet the perfection that would be ideally desirable, the good faith of family, friends and EMS personnel must be assumed for any system to work. Seeking perfection in a human designed and operated system can very well be the enemy of the good.

The second problem is the document itself. Is it legal? The answer to this question varies not only with the many subtle technicalities of legal prose, but also with the interpretation of the law and the specific jurisdiction involved. Without very specific legislative guidance, medical personnel would be uncomfortable with the legality of advance directives proffered in the prehospital environment (7). At the present time, most jurisdictions have nebulous and often conflicting statutes and common laws dealing with DNR orders, and with which individuals are able to give permission for withdrawing medical care from incompetent patients (22). As the NAEMSP noted, 'unfortunately, while many of these issues have been addressed in the inpatient setting, little work has been done to resolve the important ethical and legal issues faced in the prehospital setting' (29). Only about 21 US jurisdictions currently have a formal provision for generalised prehospital DNR orders (12). It is unknown how many EMS jurisdictions control the 
more than 17,000 EMS agencies in the US. All such guidelines may need to be re-evaluated in the light of the recent ruling of the US Supreme Court in Cruzan v Director, Missouri Department of Health (27). The court held that states may require 'clear and convincing evidence', including written advance directives, before life support may be withdrawn. It could be assumed that this will also be required for life support to be withheld.

Even if the document is legal, is it valid? What if the patient has invalidated the document; what if the prognosis has changed? Is it clear that a mistake would not be made in the presence of an old document? From a moral viewpoint, a more serious question is whether the document was obtained under duress or without informed consent. Since it is unclear whether or not inpatient DNR orders are often obtained without full disclosure and with obvious bias on the part of the clinician, it would probably be even less clear and present more potential for abuse if done outside the hospital setting. It is agreed that precautions must be incorporated into any prehospital DNR system to prevent misuse $(10,30)$.

Another problem is the potential liability for both EMS and hospital-based clinicians who would acquiesce to the request to withhold treatment that is incorporated in such a document. Any statute attempting to address this problem will need to be more specific in limiting liability than most legislatures have yet been willing to be. 'A component of most of the legislation includes a release from liability of those health workers who honour the content of the Living Will. Unfortunately, it is not clear in all states that EMT/paramedics are included in the definition of health care worker, thus opening their liability in such cases' (29). However, the Anchorage, Alaska coroner who supervises their prehospital DNR system stated that '... legal risks are easily overplayed ... unlikely legal contingencies do not warrant withholding valuable public services' (32).

Finally, what was the purpose for which the document was drafted? Was it, as would generally be supposed, signed in order to avoid a prolongation of a certain death from a terminal disease? Or was it drafted in order to assist a suicide by a mentally unstable individual? Or even more nefarious, was it drafted by potential heirs or a disgruntled spouse with the intention of having the medical community abet a murder? Ascertaining whether the purpose of such a document was within legal and moral guidelines could prove to be very difficult, if not impossible. In an operational sense, this criterion probably cannot be met.

Based on the case presented, a specific question must be raised. Which situation is the greater wrong, attempting to resuscitate a dying patient who does not wish the 'help', or not resuscitating a patient who does want these efforts? Both morally and legally, the weight would seem to favour resuscitation to protect those who may inadvertently not get the help when desired. But the reality of the EMS systems is that this is an extremely rare event. The presumption in all systems is toward resuscitation and maintaining life. In a well-functioning prehospital DNR system, resuscitation would occur in patients desiring such care unless the effort would obviously be medically futile or an error in identification is made. To avoid errors, systems already in place have stated that 'to protect the public interest procedures are designed so that any errors will lean in the direction of administrative caution' (32). The enormous burden of injustice and harm lies in erroneous resuscitation attempts. As one writer has said: 'Initiation of cardiopulmonary resuscitation when the death is the result of an endstage, irreversible, and imminently terminal illness against the patient's prior request is immoral and indefensible' (30). The AHA specifically states that " "prolongation of life", as used in the Saikewicz case, does not mean a mere suspension of the act of dying, but contemplates, at the very least, a remission of symptoms enabling a return toward a normal, functioning, integrated existence' (6). It is hard to fathom how a minuscule number of accidentally omitted resuscitations in end-of-life situations could not more than outweigh the overwhelming good that would be accomplished.

Is there a solution? Is there a system for prehospital DNR orders that would work? Yes. Some (relatively few) prehospital DNR order systems are already operational in the US. But some specific elements are necessary. First, there needs to be comprehensive and specific legislation detailing the circumstances in which such a document could be used, the wording or alternative wordings of such a document, and the absolute protection from liability for those practitioners who comply with the document. Second, there needs to be a reasonable method of identifying individuals who have a currently valid DNR order. At the same time, the currently valid document must be immediately available to the clinicians.

There are several, progressively more accurate, methods which can be used to make certain of the patient's identity and to make sure staff are aware of an existing DNR order. The common method is to rely on relatives and friends in attendance with the patient. The order (or a copy) could also be in the hospital record, with the person wearing an identification band. The order could also be kept on computer within the EMS dispatch system (the hard copies kept with the patient and at the hospital). Finally, the actual order could be kept permanently with the patient.

Both the problems of patient identification and the location of the document could be solved by a method similar to that used by the Medic Alert ${ }^{\mathrm{R}}$ and other currently used medical problem/allergy identification systems. A microfilm copy of the patient's DNR document in a tamper-proof container could be placed, on the patient, perhaps subcutaneously in the pectoral region. A standard location, perhaps over an area where defibrillation paddles would be placed, with an 
overlying tattoo, such as 'DNR', would provide practitioners with a rapid method of identification. One experienced clinician who has seen the problems perpetrated by the current system of prehospital resuscitation has had a symbol indicating not to defibrillate tattooed in just this position. Its efficacy in preventing unwanted resuscitation has yet to be tested. This, however, is too extreme for most people, and too cumbersome to work well within the EMS system. A simple method of identification with the easiest notification of the EMS personnel, similar to the systems described, seems to be adequate.

Relatively few US jurisdictions as yet have a prehospital order system, although it is an idea whose time is overdue. Society's imperative to use available technology has pushed us into a situation where a technique to save those with a potential to continue a meaningful and wanted existence is being used indiscriminately to prolong the agony of death. The pain being generated and the resources being lost due to the inability to halt a useless resuscitation in the dying is more than our society can continue to tolerate. As an interim measure, clinicians must make an extra effort to discuss the methods of death and dying with their terminal patients. Ultimately, though, we will have to bite the bullet and implement pre-hospital DNR systems in all existing EMS systems.

Kenneth $V$ Iserson MD MBA FACEP is a Senior Scholar at the Center for Clinical Medical Ethics, University of Chicago and Associate Professor of Surgery (Emergency Medicine) at the University of Arizona, Tucson.

\section{References}

(1) Younger S J. Do-not-resuscitate orders: no longer secret, but still a problem. Hastings Center report 1987; Feb: 24-33.

(2) American Heart Association. Textbook of advanced cardiac life support. Dallas, Texas: AHA, 1987.

(3) American Medical Association. Standards and guidelines for cardiopulmonary resuscitation and emergency cardiac care: VII. Medicolegal considerations and recommendations - orders not to resuscitate. Fournal of the American Medical Association 1980; 308: 716-717.

(4) Eliastam M, Duralde T, Martinez F, Schwartz D. Cardiac arrest in the emergency medical service system: guidelines for resuscitation. Fournal of the American College of Emergency Physicians 1977; 6: 525-529.

(5) Bioethics Committee, American College of Emergency Physicians. Medical, moral, legal, and ethical aspects of resuscitation for the patient who will have minimal ability to function or ultimately survive. Annals of emergency medicine 1985; 14: 919.

(6) American Heart Association. Textbook of advanced cardiac life support. Dallas, Texas: AHA, 1987: 273-277.

(7) Emergency Medical Services Committee, American College of Emergency Physicians. Guidelines for do not resuscitate orders in the prehospital setting (position paper). Dallas, Texas: ACEP, 1988.
(8) Miles S H, Crimmins T J. Orders to limit emergency treatment for an ambulance in a large metropolitan area. Fournal of the American Medical Association 1985; 254: 525-527.

(9) Iserson K V, Sanders A B, Mathieu D R, Buchanan A E. Ethics in emergency medicine. Baltimore: Williams and Wilkins, 1986, 105-157.

(10) Ayres R J, Jr. Current controversies in prehospital resuscitation of the terminally ill patient. Prehospital and disaster medicine 1990; 5,1: 49-57.

(11) Iserson K V, Rouse F. Prehospital DNR orders. Hastings Center report 1989; 19,6: 17-19.

(12) Sachs G A, Miles S H, Levin R A. Limiting resuscitation: emerging policy in the emergency medical system. Annals of internal medicine 1991; 114: 2.

(13) Maryland: hospice/EMS palliative care protocol. 1988 Mar 3.

(14) Hennepin County, MN: directives to limit emergency medical treatment. 1988 Nov.

(15) Kansas City, MO: prehospital DNR form. 1989 Mar 1.

(16) Napa County, CA: Napa County EMS do not resuscitate orders; policy no: 90-01-005/B/14. 1990 Mar 19.

(17) Anchorage, Alaska: memo from D Charlene Doris, coroner: 1984 Apr 26.

(18) Johnson County, KS: protocol for the non-viable, terminally ill, and do not resuscitate (DNR) patients. Autumn 1989.

(19) Juneau, AK. EMS do not resuscitate protocol. 1990.

(20) President's Commission for the Study of Ethical Problems in Medicine and Biomedical and Behavioral Research. Deciding to forego life-sustaining treatment: a report on the ethical, medical and legal issues in treatment decisions. Washington, DC: US Government Printing Office, 1983.

(21) President's Commission for the Study of Ethical Problems in Medicine and Biomedical and Behavioral Research. Defining death: a report on the medical, legal and ethical issues in the determination of death. Washington, DC: US Government Printing Office, 1981.

(22) Wanzer S H, Adelstein S J, et al. The physician's responsibility toward hopelessly ill patients. New England journal of medicine 1984; 310: 955-959.

(23) Bedell S E, Delbance T L. Choices about cardiopulmonary resuscitation in the hospital: when do physicians talk with patients? New England journal of medicine 1984; 309: 1089.

(24) Iserson K V. Ethics of emergency medicine. Fournal of emergency medicine 1985; 3: 161.

(25) Lee M A, Cassel C K. The ethical and legal framework for the decision not to resuscitate. Western journal of medicine 1984; 140: 117-122.

(26) Cruzan v Harmon, 760 SW 2d 408, at 419-424 (Missouri 1989) (en banc).

(27) Cruzan v Director, Missouri Department of Health, - US -, 58 LW 4916, 1990.

(28) Schloendorff v Society of New York Hospital, 211 NY 125, 105 NE 92, 1914.

(29) National Association of Emergency Medical Services Physicians (NAEMSP). Consensus document on resuscitation decisions in the prehospital setting. Special bulletin. NAEMSP, 1989.

(30) Crimmins T J. The need for a prehospital DNR system. Prehospital and disaster medicine 1990; 5,1: 47-48.

(31) Annas G J. CPR: The beat goes on. Hastings Center report 1982; 12: 24-25.

(32) Marshall L. Resuscitating the terminally ill. Fournal of the emergency medical services 1985; 10: 24-28. 